\title{
Erratum
}

\section{Hyperbolicity, Sinks and Measure in One Dimensional Dynamics}

\author{
Ricardo Mañé \\ Instituto de Matematica Pura e Aplicada, Edificio Lelio Gama, Estrada Dona Castorina, 110, CEP \\ 22, 460, Rio de Janeiro, Brasil
}

Commun. Math. Phys. 100, 495-524 (1985)

In our paper "Hyperbolicity, sinks and measure in one dimensional dynamics" (Commun. Math. Phys. 100, 495-524 (1985)), the proof of the crucial Lemma I.3 is not complete. This proof is divided in two cases depending on whether $A=N$ or $\Lambda \neq N$. The case $\Lambda=N$ is correct. The proof of the case $A \neq N$ begins by taking an open interval $U \subset \Lambda^{c}$ having an endpoint $x$ contained in $\Lambda$, and almost immediately we apply to $x$ a Lemma (Lemma II.2) that requires $x$ to be non-periodic. But a point $x$ chosen as we did can be periodic, and, even more important, it is easy to produce examples of sets $\Lambda$ satisfying the hypothesis of I.3 such that every $x \in \Lambda$ that is an endpoint of an open interval contained in $\Lambda^{c}$, is periodic. Therefore the proof must be extended in order to cover these cases. This can be done as follows, by a suitable combination of tools already developed in the paper.

We have to prove Lemma $\mathrm{I} .3$ when $\Lambda \neq N$. We shall do it through the following steps.

We have to prove Lemma I.3 when $\Lambda \neq N$. First observe that it follows trivially from the definition that $\left(J,\left\{\varphi_{n}\right\}\right)$ is a coherent sequence associated to $\Lambda$ if and only if it is a coherent sequence associated to $\bigcap_{n \geq 0} f^{n}(\Lambda)$. Follows from this remark that an interval $J$ is adapted to $\Lambda$ if and only if it is adapted to $\bigcap_{n \supseteq 0} f^{n}(J)$ because the definition of interval adapted to a set $\Lambda$ only involves the family of coherent sequences associated to $\Lambda$, that as we said, coincides with the family of coherent sequences associated to $\bigcap_{n \geq 0} f^{n}(\Lambda)$.

Therefore it suffices to prove Lemma I.3 in the surjective case, i.e. when $f(\Lambda)=A$, because, once proved in this case, if we want to prove it in the general case of a compact set $\Lambda \neq N$ satisfying only $f(\Lambda) \subset \Lambda$, we apply the surjective case to $\bigcap_{n \geq 0} f^{n}(\Lambda)$ (that obviously satisfies $f\left(\bigcap_{n \geq 0} f^{n}(\Lambda)\right)=\bigcap_{n \geq 0} f^{n}(\Lambda)$ ). This yields an interval $J$ adapted to $\bigcap_{n \geq 0} f^{n}(\Lambda)$ ) (and then, as explained above, adapted to $\Lambda$ ) satisfying the properties in Lemma I.3 for $\bigcap_{n \geqq 0} f^{n}(\Lambda)$. Since these properties only involve the family of coherent 
sequences associated to $\bigcap_{n \geq 0} f^{n}(\Lambda)$, that coincides with the family of coherent sequences associated to $\Lambda$, it follows that $J$ satisfies Lemma I.3 for $\Lambda$.

Then we have to prove I.3 when $\Lambda \neq N$ and $f(\Lambda)=\Lambda$. We shall do it through the following steps.

Step I. If an interval $J$ adapted to $\Lambda$ satisfies the following property:

(*) Either $F(J, \Lambda)=\varnothing$ or there exists $0<\lambda<1$ that $\left|\psi^{\prime}(x)\right|<\lambda$ for all $x \in J$ and $\psi \in F(J, \Lambda)$, then $J$ satisfies Lemma I.3.

This step is just Lemma II.4. We include it here because it reduces the proof of 1.3 to finding an interval $J$ adapted to $A$ and satisfying (*).

Step II. If there exists an open interval $U \subset \Lambda^{c}$ having an endpoint $x \in \Lambda$ that is not a periodic point, then there exists an interval $J$ adapted to $\Lambda$ satisfying $(*)$.

This step is what is correctly and explicitly proved in the proof of I.3 when $\Lambda \neq N$ (page 519).

Step III. Let $J_{0}$ be an interval adapted to $\Lambda$ such that for all $\varepsilon>0$ there exists $\psi_{0} \in F\left(J_{0}, \Lambda\right)$ satisfying diam $\psi_{0}\left(J_{0}\right)<\varepsilon$, then there exists an interval $J$ adapted to $\Lambda$ satisfying $(*)$.

The proof of this property is contained in the proof of 1.3 in the case $A=N$. In fact, to prove I. 3 when $A=N$, we use $A=N$ to show that there exists an interval $J_{0}$ satisfying the hypothesis of Step III, and then (without using again the hypothesis $\Lambda=N$ ) we prove that this hypothesis implies the existence of $J$ satisfying $(*)$.

Step IV. If there exists an interval $J_{0}$ adapted to $\Lambda$ such that $F\left(J_{0}, \Lambda\right)$ contains infinitely many maps, then there exists an interval $J$ adapted to $\Lambda$ satisfying $(*)$.

To prove this property observe that $\Sigma$ diam $\psi\left(J_{0}\right)<\operatorname{diam}\left(J_{0}\right)$, where the sum is taken over all the maps $\psi \in F\left(J_{0}, \Lambda\right)$. Then, if $\# F\left(J_{0}, \Lambda\right)=\infty$, there exists $\psi_{0} \in F\left(J_{0}, \Lambda\right)$ with diam $\psi\left(J_{0}\right)$ arbitrarily small, thus proving that $J_{0}$ satisfies the hypothesis of Step III.

Given a set $J \subset N$ and $x \in J$ define $N(x, J)$ as the minimum $n>0$ such that $f^{n}(x) \in J$. If $f^{n}(x) \notin J$ for all $n>0$ set $N(x, J)=+\infty$.

Step $V$. If $J_{0}$ is an interval adapted to $\Lambda$ such that $\sup \left\{N\left(x, J_{0}\right) \mid x \in \Lambda \cap J_{0}\right\}=\infty$, then there exists $J$ satisfying $(*)$.

First suppose that there exists a sequence $\left\{x_{i}\right\} \subset J_{0} \cap A$ such that $n_{i}=N\left(x_{i}, J_{0}\right)<$ $\infty$ for all $i$ and $\lim _{i \rightarrow+\infty} N\left(x_{i}, J_{0}\right)=\infty$. We can assume that $n_{i} \neq n_{j}$ if $i \neq j$. Then the maps $\psi_{i} \in F\left(J_{0}, \Lambda\right)$ with $\psi_{i}\left(f^{n_{i}}\left(x_{i}\right)\right)=x_{i}$ are all different. Hence $\# F\left(J_{0}, \Lambda\right)=\infty$ and the existence of $J$ follows from Step IV. Now suppose that there exists $x \in J_{0} \cap \Lambda$ with $N\left(x, J_{0}\right)=\infty$. When $x$ is accumulated by points $y \in J_{0} \cap A$ with $N\left(y, J_{0}\right)<\infty$, then, since $N(x, J)=\infty$, there exists a sequence $\left\{x_{i}\right\}$ satisfying the hypothesis of the previous case. When every $y \in J_{0} \cap \Lambda$ nearby $x$ satisfies $N\left(y, J_{0}\right)=\infty$, then there exists an interval $J \subset J_{0}$ adapted to $\Lambda$, containing $x$, such that $N(y, J)=\infty$ for all $y \in \Lambda \cap J$. This means that $F(J, \Lambda)=\varnothing$ and then (*) is satisfied.

Step VI. If there exists $x \in \Lambda$ such that $x \notin \omega(x)$, then there exists $J$ satisfying $(*)$.

Suppose that $x \in \Lambda$ satisfies $x \notin \omega(x)$. Take (by II.1) an interval $J_{0}$ adapted to $\Lambda$ 
containing $x$ and so small that $f^{n}(x) \notin J_{0}$ for all $n>0$. Then $N\left(x, J_{0}\right)=\infty$ and Step VI follows from $\mathrm{V}$.

Step VII. If there exists a sequence of periodic points $x_{i} \in \Lambda$ with periods $n_{i} \rightarrow+\infty$ and $\left\{x_{i}\right\}$ converging to a periodic point $x$, then there exists $J$ satisfying $(*)$.

Let $\gamma$ be the orbit of $x$. The existence of the sequence $\left\{x_{i}\right\}$ with periods $n_{i} \rightarrow+\infty$ implies that there exists $y \notin \gamma$ such that $f(y) \in \gamma$ and the orbits of the points $x_{i}$ accumulate in $y$. Hence $y \in \Lambda$. Obviously $\omega(y)=\gamma$. Since $y \notin \gamma$, we obtain $y \notin \omega(y)$ that by Step VI implies the existence of $J$ satisfying (*).

Step VIII. Let $\Lambda_{1}$ be the closure of the non-periodic points in $\Lambda$. If there exists a source $x \in \Lambda-\Lambda_{1}$, then there exists $J$ satisfying (*).

Suppose first that $x \notin \overline{\Lambda-\{x\}}$. Then we can take an open interval $U$ containing $x$ and such that, if $n$ is the period of $x, f^{n}$ maps $U$ diffeomorphically onto $f^{n}(U) \supset U$ and $f^{n}(U) \cap A=\{x\}$. Then $J=f^{n}(U)$ is obviously adapted to $\Lambda$ and $F(J, \Lambda)$ contains only one element, namely the map $\psi: J \rightarrow U$ such that $\psi(x)=x$ and $f^{n} \psi(y)=y$ for all $y \in J$. Then $J$ satisfies $(*)$. Now suppose that $x$ is accumulated by points in $\Lambda-\{x\}$. Since $x \notin \Lambda_{1}$ these points must be periodic, and since $x$ is a source their periods must go to $\infty$ when they approach $x$. Then we fall in the hypothesis of Step VII.

Step IX. If there exists a periodic point $x \in \Lambda_{1}$, then there exists $J$ satisfying $(*)$.

Suppose that $\gamma$ is the orbit of $x$. If there exists $y \in \Lambda$ such that $f(y) \in \gamma$ and $y \notin \gamma$, then $y \notin \omega(y)=y$ and by Step VI we are done. Now suppose that there is no such $y$. There exists an arbitrarily small neighborhood $U$ of $\gamma$ and a continuous map $\varphi: U \rightarrow N$ such that $\varphi / \gamma=(f / \gamma)^{-1}$ and $f \varphi(x)=x$ for all $x \in U$. The absence of points $y \in A-\gamma$ with $f(y) \in \gamma$ implies that if $U$ is small enough then

$$
f^{-1}(\{p\}) \cap \Lambda=\varphi(p)
$$

for all $p \in U \cap \Lambda$. Now take a non-periodic point $z \in \Lambda$ so close to $x$ that for some $m>0$ the interval $(x, z)$ is mapped diffeomorphically onto $\left(x=f^{m}(x), f^{m}(z)\right)$ and $\left(f^{j}(x), f^{j}(z)\right) \subset U$ for all $0 \leqq j \leqq m$. When $f^{m}(z)<z$ the $\omega$-limit of $z$ is a periodic orbit in $U$, and since $z$ is not periodic we have $z \notin \omega(z)$, and by VI we are done. When $f(z)>z$ we claim that $z$ is not recurrent. Again by VI, this proves what we want. To prove the claim, suppose that $z$ is recurrent. Then we can take $N>n$ such that $f^{N}(z) \in\left(x, f^{m}(z)\right)$. Define $V=\bigcup_{0}^{m}\left(f^{j}(x), f^{j}(z)\right)$. Then $V \subset U$, and it is easy to check (using that $f^{m}(z)>z$ ) that

$$
\varphi(V) \subset V .
$$

Moreover $f^{N}(z) \in V \cap \Lambda$. By $(* * *)$ and $(* *)$ :

$$
f^{N-1}(z)=\varphi f^{N}(z) \in \varphi(V) .
$$

Continuing this argument we conclude that $f^{j}(z) \in V$ for all $0 \leqq j \leqq N$. But then $z$ has all its orbit contained in $V$ because $N$ can be taken arbitrarily large. Then $z$ cannot be recurrent, thus completing the proof of the claim and Step IX.

Now let us prove Lemma I.3 when $A \neq N$. Take an open interval $U \subset \Lambda_{1}^{c}$ having 
an endpoint $x_{0} \in \Lambda_{1}$. When $x_{0}$ is periodic, Lemma I.3 follows from Step IX and I. When $x_{0}$ is not periodic we apply Step II to $\Lambda_{1}$ and we obtain an interval $J$ adapted to $\Lambda_{1}$ such that either $F\left(J, \Lambda_{1}\right)=\varnothing$ or there exists $0<\lambda<1$ satisfying $\left|\psi^{\prime}(x)\right|<\lambda$ for all $x \in J$ and $\psi \in F\left(J, \Lambda_{1}\right)$. Take a non-periodic point $x_{1} \in \Lambda_{1} \cap J$ (recall that the nonperiodic points are dense in $\Lambda_{1}$ ). Suppose first that $\Lambda-\Lambda_{1}$ doesn't accumulate at $x_{1}$. Then (by II.2) we can take an interval $J_{0} \subset J$ adapted to $\Lambda_{1}$ containing $x_{1}$, and so small that $J_{0} \cap\left(\Lambda-\Lambda_{1}\right)=\varnothing$. This means that $J_{0} \cap \Lambda=J_{0} \cap \Lambda_{1}$ and then $F\left(J_{0}, \Lambda_{1}\right)=$ $F\left(J_{0}, \Lambda\right)$. Then, if $\psi \in F\left(J_{0}, \Lambda\right)$, it follows that $\psi \in F\left(J_{0}, \Lambda_{1}\right)$. It is easy to see that this implies that $\psi$ can be written as a composition of elements of $F\left(J, \Lambda_{1}\right)$. Hence $\left|\psi^{\prime}(x)\right|$ $<\lambda$ for all $x \in J_{0}$. We have thus checked that either $F\left(J_{0}, \Lambda\right)=\varnothing$ or $\left|\psi^{\prime}(x)\right|<\lambda$ for all $x \in J_{0}$ and $\psi \in F\left(J_{0}, \Lambda\right)$. According to Step I, this proves Lemma I.3. Now suppose that $\Lambda-\Lambda_{1}$ accumulates at $x_{1}$. Take a sequence of points $x_{i} \in \Lambda-\Lambda_{1}$ (that by definition of $A_{1}$ must be periodic) converging to $x_{1}$. We claim that if $\gamma_{i}$ is the orbit of $x_{i}$, then for all $\varepsilon>0$ there exists $i_{0}$ such that $\gamma_{i} \subset B_{\varepsilon}\left(\Lambda_{1}\right)=\left\{z \mid d\left(z, \Lambda_{1}\right)<\varepsilon\right\}$ for all $i \geqq i_{0}$. To prove this first observe that the periods of $x_{i}$ must go to $\infty$ when $i \rightarrow+\infty$. because they converge to a non-periodic point $x_{1}$. Then, if the claim were false, there would exist points $y_{i} \in \gamma_{i}$, for arbitrarily large values of $i$, converging to a point $y \notin \Lambda_{1}$. Then $y$ is periodic (because it doesn't belong to $\Lambda_{1}$ ) and it is a limit of a sequence of periodic points whose periods go to infinity. Hence, by Step VII, Lemma I.3 is proved. Therefore we have only to prove Lemma I.3 when the claim is true. Now take an interval $J_{1} \subset \bar{J}_{1} \subset J$ adapted to $\Lambda$ and containing $x_{1}$ (whose existence is granted by II.2). Set $\delta=d\left(J_{1}, J^{c}\right)$. We can assume that $N_{0}=\sup$ $\left\{N\left(x, J_{0}\right) \mid x \in J_{0} \cap A\right\}<\infty$, because otherwise we are done by Step $\mathrm{V}$. Choose $0<\varepsilon<\delta / 2$ such that if $a \in \Lambda_{1}$ and $y \in N$ satisfy $d(y, a)<\varepsilon$, then $d\left(f^{j}(a), f^{j}(y)\right)<\delta / 2$ and the interval $\left(f^{j}(a), f^{j}(y)\right)$ doesn't contain critical points for all $0 \leqq j \leqq N_{0}$. Now take a point $x_{i}$ such that its orbit is contained in $B_{\varepsilon}\left(\Lambda_{1}\right)$. We shall prove that for every $x \in \gamma_{i} \cap J_{1}$ there exists $n>0$ satisfying $f^{n}(x) \in J_{1}$ and $\left|\left(f^{n}\right)^{\prime}(x)\right|>1 / \lambda$. This easily implies that $\gamma_{i}$ is a source, and since this source is not contained in $\Lambda_{1}$, we are done by Step VIII. Suppose that $x \in \gamma_{i} \cap J_{1}$. There exists $0<n \leqq N_{0}$ such that $f^{n}(x) \in J_{1}$. Since $\gamma_{i} \in B_{\varepsilon}\left(\Lambda_{1}\right)$ and $0<\varepsilon<\delta / 2$, there exists $a \in \Lambda_{1}$ such that $d(a, x)<\varepsilon<\delta / 2$. Hence $a \in \Lambda_{1} \cap J$. Moreover $d\left(f^{n}(a), f^{n}(x)\right)<\delta / 2$. Since $f^{n}(x) \in J_{0}$, it follows that $f^{n}(a) \in J$, and then $f^{n}(a) \in J \cap \Lambda_{1}$. Denote $F^{\prime}\left(J, \Lambda_{1}\right)$ the set of maps obtained as compositions of maps in $F\left(J, \Lambda_{1}\right)$. Then there exists $\psi \in F^{\prime}\left(J, \Lambda_{1}\right)$ with $\psi\left(f^{n}(a)\right)=a$. Since the interval $\left(f^{j}(a), f^{j}(x)\right)$ doesn't contain critical points for all $0 \leqq j \leqq n$, it is easy to check $\psi\left(f^{n}(x)\right)=x$. Hence $\left|\left(f^{n}\right)^{\prime}(x)\right|=\left|\psi^{\prime}\left(f^{n}(x)\right)\right|^{-1}>1 / \lambda$.

Communicated by J.-P. Eckmann

Received April 22, 1987 